\title{
P-0664 Genetic Associations with Diabetic Retinopathy and Coronary Artery Disease in Emirati Patients with Type-2 Diabetes Mellitus
}

\author{
Sarah K. Azzam¹, Wael Osman², Sungmun Lee', Kinda Khalaf ${ }^{1}$, Ahsan Khandoker', Wael \\ Almahmeed ${ }^{3}$, Herbert Jelinek ${ }^{4}$, Habiba Al Safar ${ }^{1 / 2}$ \\ 1 Biomedical Engineering Department, Khalifa University of Science and Technology, Abu Dhabi, UAE \\ 2 Khalifa University Center of Biotechnology, Abu Dhabi, UAE \\ 3 Heart and Vascular Institute, Cleveland Clinic, Abu Dhabi, UAE \\ 4 School of Community Health, Charles Sturt University, Albury, Australia

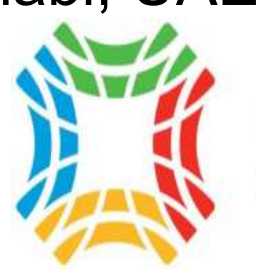 \\ KHAL̈IFA UNIVERSITY்
}

BACKGROUND

Diabetes is one of the largest health emergencies globally, where the International Diabetes Federation (IDF) reported that 415 million people have been diagnosed with diabetes in 2015 , and the number is expected to rise to 642 million by 2040 [1]. In the United Arab Emirates (UAE), diabetes prevalence in adults between the ages of 20 to 79 years old, was found to be $19.3 \%$ in 2015. Type 2 diabetes mellitus (T2DM) accounts for more than $90 \%$ of patients diagnosed with diabetes; characterizing T2DM as the most common type of diabetes [2].

\section{AlMS}

Long-term debilitating complications and health issues are consequent events to T2DM. Microvascular complications such as diabetic retinopathy (DR), and macrovascular complications such as coronary artery disease (CAD), are examples of T2DM-associated complications. The development of these two complications is affected by genetic risk factors. A case-control study was performed, focusing on investigating genetic variations in association with DR and CAD, in T2DM patients from the UAE.

\section{METHODS}

A total of 407 Emirati patients with T2DM were recruited into the current study. Study population was categorized based on the presence or absence of DR and CAD. For association analyses, we selected 17 Single Nucleotide Polymorphisms (SNPs) that were reported in previous studies. In evaluating the association between the 17 SNPs and $\mathrm{DR}, \mathrm{CAD}$, or both complications, a multivariate logistic regression test was conducted using PLINK software [3], with a significance level set at $p<0.05$.

\section{RESULTS}

Demographic data, clinical and laboratory data of participants were analyzed. For category 1 , patients diagnosed with DR had significantly higher age and a higher prevalence of dyslipidemia as compared to controls without DR. In category 2, T2DM patients with CAD had also significantly higher age with higher total cholesterol, LDL-cholesterol, hypertension, and presence dyslipidemia. Significantly more male patients presented with CAD and indicated longer smoking history. For category 3 , patients with both conditions had similar results to category 2.

The SNPs rs9362054 near the CEP162 (centrosomal protein 162) gene and rs4462262 near the UBE2D1 (ubiquitin conjugating enzyme E2 D1) gene were significantly associated with DR and rs12219125 nearby the $P L X D C 2$ (Plexin domain-containing protein 2) gene was significantly associated with CAD. Moreover, rs9362054 nearby the CEP162 gene was significantly associated with both complications. Of note is that $K I A A 0825$ gene, a protein-coding gene with uncharacterized protein KIAA0825, might have a protective role either directly or in linkage with other genes. The results of associations between SNPs and T2DM complications (DR and CAD) considering age, dyslipidemia, hypertension, total cholesterol and LDL-cholesterol as covariates are shown in Table 1.

Table 1. Results of significant associations between tested SNPs and T2DM complication: DR, CAD, or a combination of both.

\begin{tabular}{lccccccc}
\hline \multicolumn{1}{c}{ SNP ${ }^{\dagger}$ ID } & Gene(s) & Chr : BP & A1/A2 & $\begin{array}{c}\text { MAF } \\
\text { Cases }\end{array}$ & $\begin{array}{c}\text { MAF } \\
\text { Controls }\end{array}$ & OR (Cl 95\%) * & P-value \\
\hline DR vs. no DR & & & & & & & \\
rs9362054 & LINC01611 & $6: 85178268$ & A/G & $39 \%$ & $50 \%$ & $1.73(1.29-2.32)$ & $\mathbf{0 . 0 0 0 2 9}$ \\
rs4462262 & LOC105378313; & $10: 59189178$ & A/G & $39 \%$ & $46 \%$ & $1.33(1.00-1.75)$ & $\mathbf{0 . 0 4 5}$ \\
CAD vs. no CAD & LOC105378314 & & & & & & \\
rs12219125 & PLXDC2 & 10: 20593087 & A/C & $3.8 \%$ & $6.3 \%$ & $2.26(1.06-4.81)$ & $\mathbf{0 . 0 3 4}$ \\
DR + CAD vs. no DR \& no CAD & & & & & & \\
rs17376456 & KIAA0825; & 5: 93557702 & G/A & $14 \%$ & $9.9 \%$ & $0.423(0.191-0.935)$ & $\mathbf{0 . 0 3 4}$ \\
rs9362054 & LOC105379087 & 6: 25178268 & A/G & $36 \%$ & $50 \%$ & $2.26(1.35-3.78)$ & $\mathbf{0 . 0 0 2 0}$
\end{tabular}

tDR, Diabetic Retinopathy; CAD, Coronary Artery Disease

${ }^{*} \mathrm{~A} 2$ was used as a reference allele.

The susceptibility genes for CAD (PLXDC2) and DR (UBE2D1) have a role in angiogenesis and neovascularization (figures 1 and 2 respectively). REFERENCES

[1] IDF Diabetes Atlas - 7th Edition. International Diabetes Federation (IDF)-Diabetes Atlas 7th Edition 2015 [2] Widmaier, E. P., Raff, H., Strang, K. T. \& Vander, A. J. Vander's human physiology: the mechanisms of body function. 581-583 (McGraw-Hill, 2011).

[3] Purcell, S. et al. PLINK: a tool set for whole-genome association and population-based linkage analyses. Am. J. Hum. Genet. 81, 559-575 (2007)
Moreover, association in terms of retinal neural processing was found between the ciliary gene CEP162 and DR, confirming previous reports (figure 3). $\quad$ rs12219125

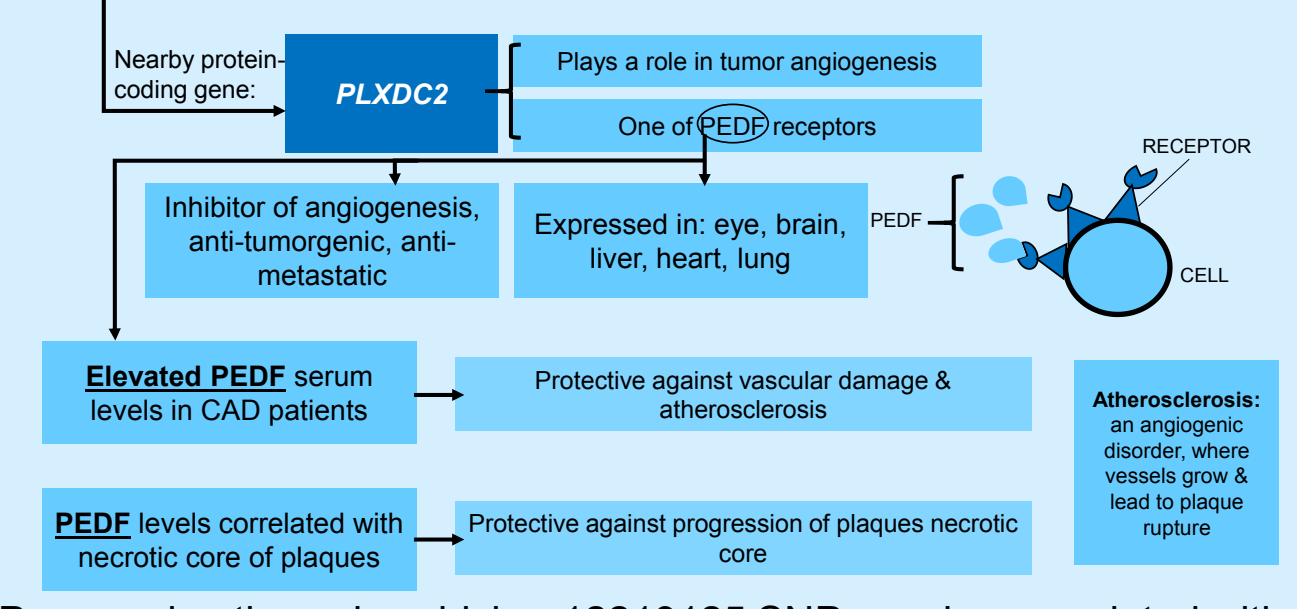

Figure 1. Proposed pathway by which rs 12219125 SNP may be associated with CAD as a

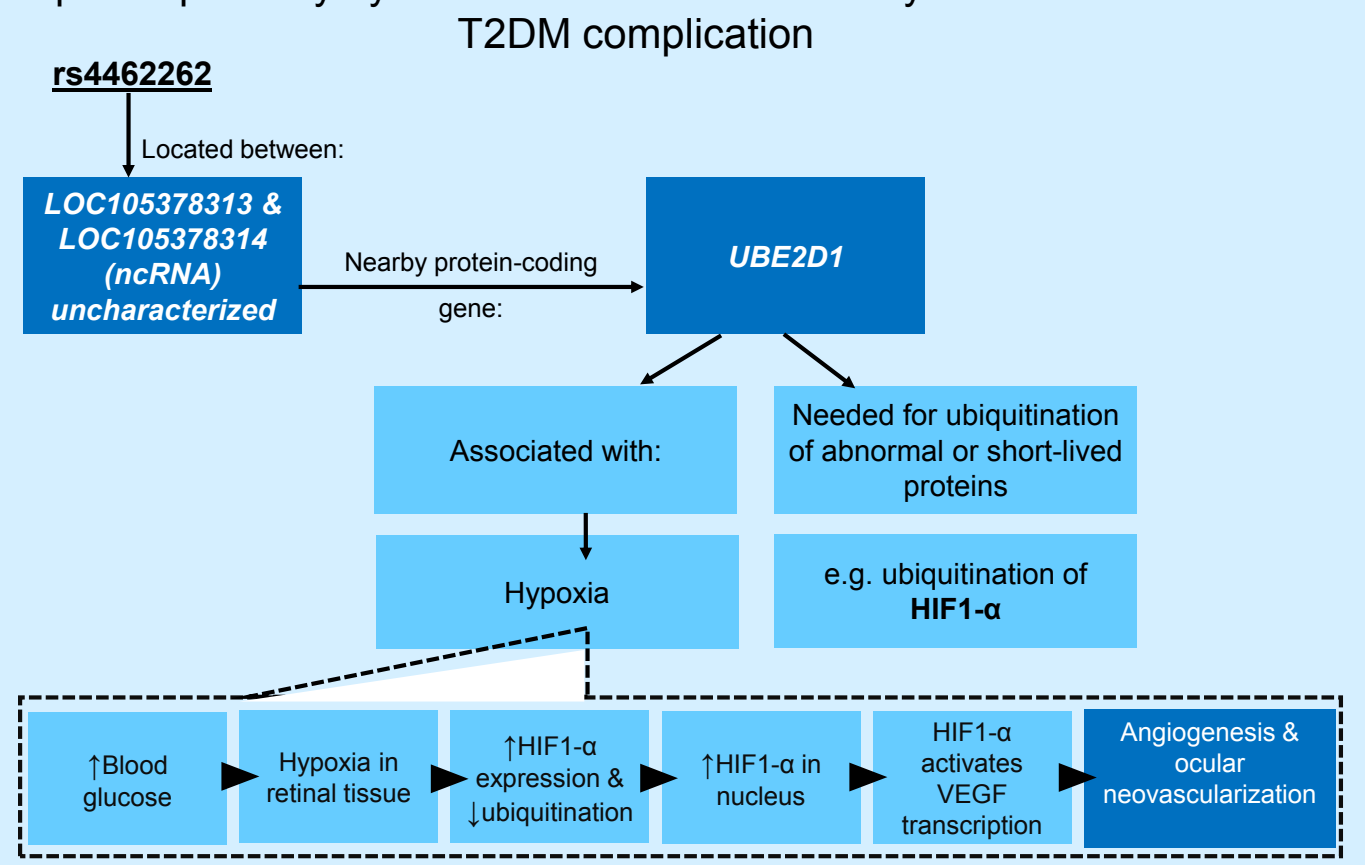

Figure 2. Proposed pathway illustrating association of rs4462262 SNP with DR

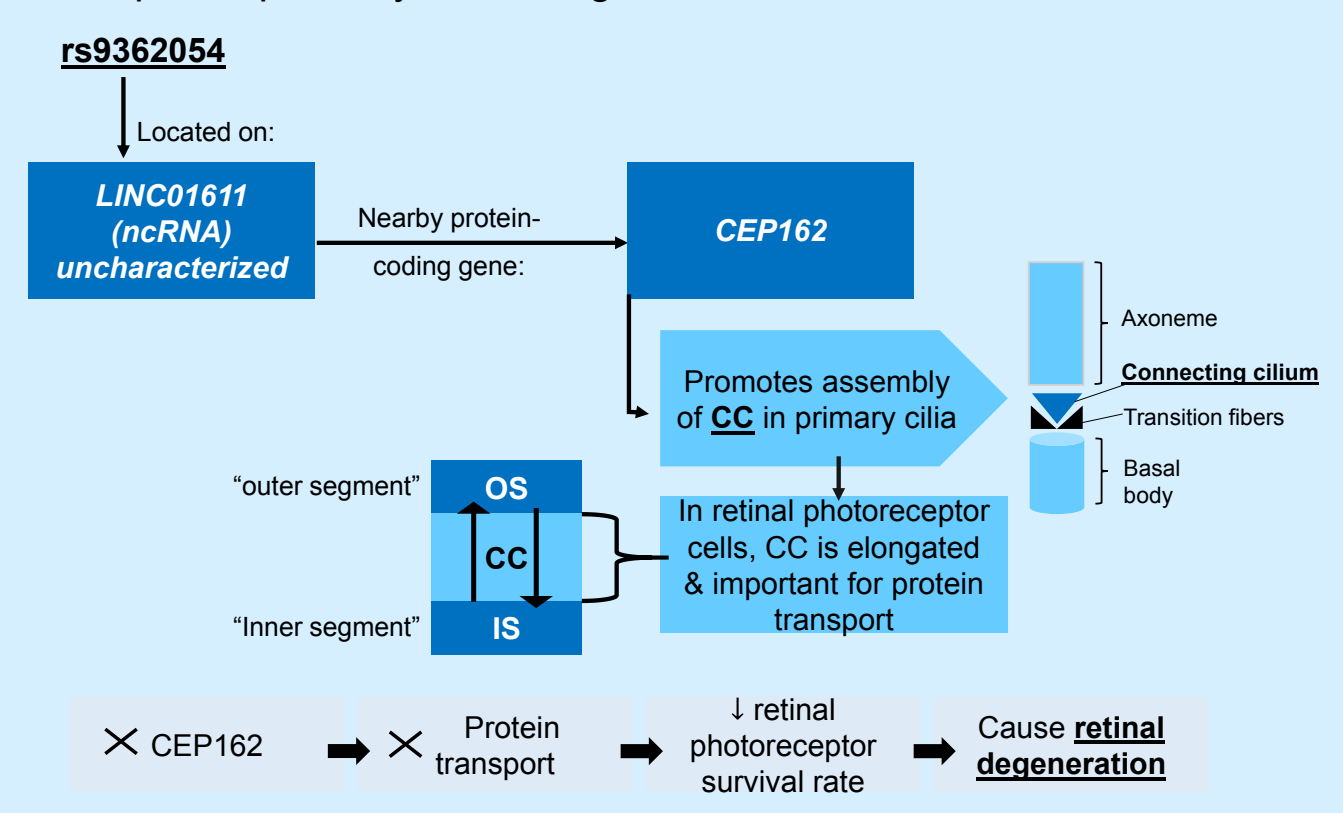

Figure 3. Proposed pathway by which rs9362054 SNP can be associated with DR CONCLUSIONS

The present study reports significant associations of different genetic loci with $D R$ and CAD. We report new associations between CAD and $P L X D C 2$, and DR with UBE2D1 using data from patients with T2DM from the UAE. 